\title{
Ionic Detoxification Reduces Obesity and Fatty Liver in Rats Fed a High- Fat Diet
}

\author{
Hsien-Tsung Yao* \\ Department of Nutrition, China Medical University, 91, Hsueh-Shih Road, Taichung, 404, Taiwan
}

\begin{abstract}
Background: Ionic detoxification (ID) or ionic water bath treatment is a simple alternative method to improve health but lacks scientific evidence. It is hypothesized that regular ID treatment can increase the excretion of toxic molecules from the body and improve metabolic disorders including obesity.

Methods: To investigate the effect of ID treatment on high-fat diet-induced obesity and fatty liver, male Wistar rats were fed a low-fat control diet, a high-fat (HF) diet, or a HF diet with ID treatment (3 times/week) for 11 weeks. Triglyceride contents and fatty acid metabolic genes in perirenal adipose tissue and liver were determined.

Results: Rats fed the HF diet had significantly higher body weight, liver weight, and adipose tissue (perirenal and epididymal) weight than did animals fed the low-fat diet. ID treatment significantly reduced body weight and perirenal adipose tissue weight induced by the HF diet. In addition, ID treatment reduced hepatic cholesterol and triglyceride contents. In liver, ID treatment reduced fatty acid synthesis (acetyl-CoA carboxylase and fatty acid synthase mRNA) more significantly than fatty acid oxidation (carnitine palmitoyl-transferase 1 and acyl CoA oxidase mRNA). In perirenal adipose tissue, ID treatment increased the lipolysis rate and reduced the triglyceride level. However, ID treatment had no significant effects on lipogenesis gene expression or adipose size in perirenal adipose tissue.
\end{abstract}

Conclusions: The results of this study suggest that regular ID treatment may have beneficial effects on reducing obesity and fatty liver.

Keywords: Ionic detoxification; Ionic water bath; Obesity; Fatty liver; High-fat diet; Rats

Abbreviations: ACC: Acetyl-CoA Carboxylase; ACOX: Acyl CoA Oxidase; ALT: Alanine Aminotransferase; CPT-1: Carnitine PalmitoylTransferase 1; FAS: Fatty Acid Synthase; HOMA: Homeostasis Model Assessment; ID: Ionic Detoxification; ORP: Oxidation Reduction Potential; PPAR- $\alpha$ : Peroxisome Proliferator-Activated Receptor $\alpha$; SREBP-1: Sterol Regulatory Element-Binding Transcription Factor 1

\section{Introduction}

Humans are now regularly exposed to electromagnetic pollution from indoor electrical equipment and outdoor electrical appliances. Studies suggest that exposure of the human body to electromagnetic pollution can lead to electrical imbalances and the production of electrostatic forces in the body and may be responsible for reducing physical activity, oxygen uptake, and immune system function and potentially increasing stress and some degenerative illnesses [1]. Recent evidence suggests that exposure to electromagnetic fields (e.g., high-frequency voltage transients, dirty electricity) is contributing to the worldwide epidemics of obesity and diabetes [2]. Also, excess accumulation of small positively or negatively charged particles (or molecules) in the body derived from exogenous electromagnetic stimulation or endogenous electrostatic forces may attract charged molecules in some tissues, resulting in the development and progression of diseases such as cardiovascular disease [3]. The electron charge in the body may remain until it can be reduced by means of an electrical current or electrical discharge.

Ionic detoxification (ID), also called ionic water bath treatment, is bathed the local or whole body in warm water with electrolysis of water and considered to be a simple way to improve health in many countries, but rigorous scientific evidence is lacking. The hands, feet, or body are bathed in warm water while an ion generator (a stainless steel spring) produces a stream of positive $(\mathrm{H}+)$ and negative $(\mathrm{OH}-)$ ions from the cathode and anode, respectively. The ion streams may attract oppositely small charged molecules to the ion generator and thus balance electricity in the body. Therefore, by continuously transmitting a microcurrent through the entire body for a period a time, ID treatment may reduce the accumulation of excessively small charged molecules in the body. After ID treatment, the amount of precipitates (neutralized molecules) can be observed in the water as various colors and unfavorable flavors from different individuals. However, it is still unclear what kinds of molecules are excreted from the body during ID treatment.

In addition to oxygen gas, abundant hydrogen gas is produced in the water (hydrogen-rich water) during electrolysis and lowers the oxidation-reduction potential (ORP). The hydrogen-rich water bath may provide electricity to the body and lower the body's electric potential similar to the action of grounding. Grounding is known to reduce the alternating current electric potential at the surface of the body and lower perturbations in electric charges of molecules inside the body [4]. Grounding can normalize the function of all body systems and improve sleep, relieve chronic pain, increase the respiratory rate and blood oxygenation, and reduce blood glucose, red blood cell aggregation, and inflammation $[4,5]$.

${ }^{*}$ Corresponding author: Hsien-Tsung Yao, Department of Nutrition, China Medical University, 91 Hsueh-Shih Road, Taichung 404, Taiwan. Tel: 886422053366; Fax 886-4-22062891; E-mail: htyao@mail.cmu.edu.tw

Received August 31, 2017; Accepted September 16, 2017; Published September 22, 2017

Citation: Hsien-Tsung Y (2017) lonic Detoxification Reduces Obesity and Fatty Liver in Rats Fed a High-Fat Diet. Altern Integ Med 5: 246. doi: 10.4172/23275162.1000246

Copyright: @ 2017 Hsien-Tsung Y. This is an open-access article distributed unde the terms of the Creative Commons Attribution License, which permits unrestricted use, distribution, and reproduction in any medium, provided the original author and source are credited. 
We hypothesized that ID treatment may normalize cell function by reducing electrical potential and electrical imbalances in the body, which may increase cell detoxification and improve metabolic disorders. In this study, we investigated the possible anti-obesity effect of ID treatment in a diet-induced obesity model, which mimics the pathogenesis of human obesity [6].

\section{Materials and Methods}

\section{Ionic detoxification (ID)}

The ID system, which included a main engine and a stainless steel spring array (ion generator), was purchased from Health and Medical Sales, Inc (USA). The salt $(\mathrm{NaCl})$ concentration in the water bath was $0.04 \%$. The microcurrent was approximately at $1 \mathrm{~A}$. The temperature of the water bath was set at $32^{\circ} \mathrm{C}$. For this study, rats were fixed in a plastic bag and each rat tail was bathed in the ID system for $8 \mathrm{~min}$, as plotted in Figure 1. The concentration of dissolved molecular hydrogen in the water bath during electrolysis was measured with an ENH-1000 electrode (TRUSTLEX Inc, Osaka, Japan). The ORP value was determined by use of an MP-103 electrode (Gondo Electronic Co., Ltd. Taipei, Taiwan). The concentration of molecular hydrogen and the ORP value in water bath during electrolysis were monitored with gentle stirring.

\section{Animals and treatment}

Ten-week-old male Wistar rats (BioLASCO Taiwan Co., Ltd., Ilan, Taiwan) were randomly divided into three groups: (a) the control group (Control), which was fed a standard laboratory rodent diet; (b) the highfat diet group (HF), which was fed a high-fat diet; and (c) the high-fat diet group with ionic detoxification (HF+ID), which was fed the highfat diet and underwent ID treatment. The high-fat diet contained $17 \%$ lard, $2 \%$ soybean oil, $0.5 \%$ cholesterol, $0.05 \%$ cholic acid, and $80.45 \%$ standard laboratory rodent diet. For ID treatment, rats were fixed in a plastic bag and each rat tail was bathed in warm water in the ID apparatus for $8 \mathrm{~min}$ (at 15:00 pm). The same treatment was conducted three times (Monday, Wednesday, Friday) weekly. After ID treatment, the ammonia concentration in bathed water was measured (Randox Laboratories, Crumlin, UK), and then the bathed water was heated in an oven at $100^{\circ} \mathrm{C}$ to remove the moisture. The residue was extracted with ethanol and the resulting extract was vacuumed dry as the lipid content of residue.

The rats were housed in plastic cages in a room kept at a temperature of $23 \pm 1^{\circ} \mathrm{C}$ and relative humidity of $60 \pm 5 \%$ with a $12 \mathrm{~h}$ light-dark cycle. Food and drinking water were available ad libitum for 11 weeks. Body weight was measured once per week. At the end of the 11 week experimental period, food intake was measured and feces were collected for 2 days. Rats were fasted for $12 \mathrm{~h}$ before being sacrificed (at 8:00 AM). The rats were sacrificed by exsanguination via the abdominal aorta while under carbon dioxide $\left(70 \% / 30 \%, \mathrm{CO}_{2} / \mathrm{O}_{2}\right)$ anesthesia. Heparin was used as the anticoagulant. Plasma was separated from the blood by centrifugation $(1750 \mathrm{xg})$ at $4^{\circ} \mathrm{C}$ for $20 \mathrm{~min}$. The liver and adipose tissues (epididymal and perirenal) from each animal were excised and weighed. In this study, we found that perirenal fat pads were reduced by ID treatment more significantly than epididymal adipose tissue weight. Therefore, epididymal adipose tissue was used for further histological and biochemical examinations. Parts of the liver and perirenal fat pads were excised and fixed in $10 \%$ neutral formalin followed by dehydration in ascending grades of alcohol, clearing in xylene, and embedding in paraffin wax. Liver sections and perirenal fat pads were stained with hematoxylin and eosin (H\&E) for the histological examination [7]. The other parts of liver and adipose tissues were immediately stored and frozen at $-80^{\circ} \mathrm{C}$.

This study was approved by the Animal Center Management Committee of China Medical University. The animals were maintained in accordance with the guidelines for the care and use of laboratory animals as issued by the Animal Center of the Ministry of Science and Technology, Taiwan.

\section{Determination of plasma biochemical parameters}

Plasma concentrations of glucose, total cholesterol, and triglyceride were determined by enzymatic methods with kits purchased from Audit Diagnostics (Cork, Ireland). Plasma insulin was determined by using a rat insulin enzyme-linked immunosorbent assay (ELISA) kit purchased from Mercodia (Uppsala, Sweden). High-density lipoprotein (HDL) cholesterol was measured after the removal of very-low-density lipoprotein and low-density lipoprotein cholesterol by heparinmanganese precipitation (Randox Laboratories, Crumlin, UK). Plasma alanine aminotransferase (ALT) activity was determined by using a commercial kit purchased from Randox Laboratories (Antrim, UK).

The homeostasis model assessment (HOMA) was used as an index of insulin resistance [8]. It was calculated by the formula

$\mathrm{HOMA}=$ fasting glucose $[\mathrm{mmol} / \mathrm{L}] \times$ fasting insulin $[\mu \mathrm{U} / \mathrm{mL}] / 22.5$.

\section{Determination of liver, adipose tissue, and fecal lipid contents}

Total lipids from liver, adipose tissue (perirenal), and feces were extracted with chloroform/methanol solution ( $/ / \mathrm{v}, 2: 1)$ according to the method of Folch et al. [9]. Triglyceride and cholesterol in the solvent extract were emulsified by the addition of Triton X-100 [10] and their contents were determined by use of commercial enzymatic kits (Randox Ltd, Antrim, UK).

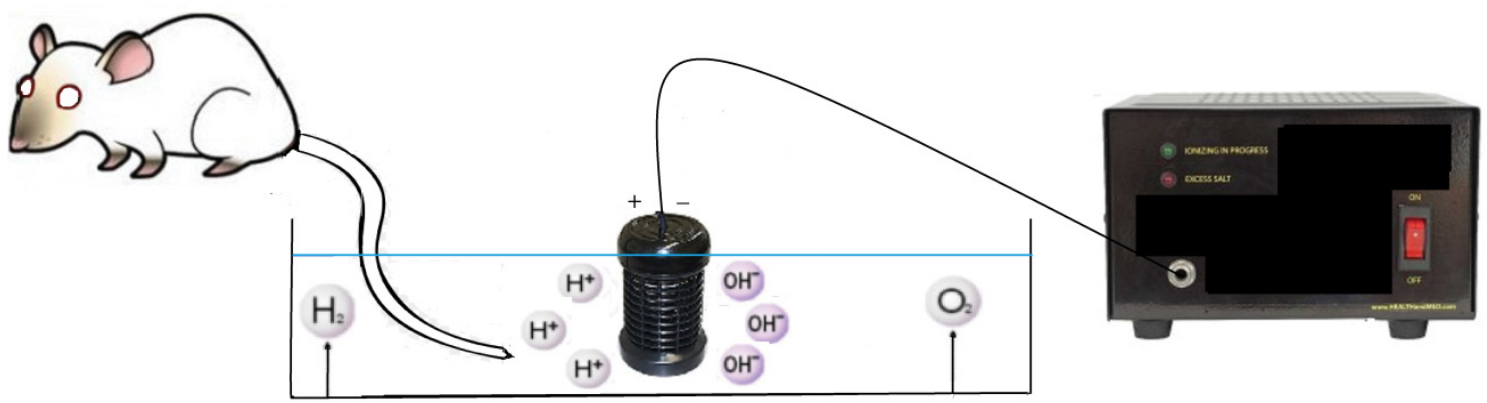

Figure 1: Rat was bathed in ID system with electrolysis of water. 


\section{Determination of fatty acid metabolic genes}

Total RNA was extracted from homogenized liver tissue by using TRIZOL reagent (Invitrogen, Carlsbad, CA, USA) according to the manufacturer's instructions. Total RNA $(1 \mu \mathrm{g})$ was reversetranscribed into first-strand cDNA by using 200 units of MMLV-RT (Promega) in a total volume of $20 \mu$ l. For real-time PCR, a SYBR system with self-designed primers and $12.5 \mathrm{ng}$ cDNA was used. The selfdesigned primers were as follows: peroxisome proliferator-activated receptor ([PPAR- $\alpha]$ forward: GGCTCGGAGGGCTCTGTCATC; reverse: ACATGCACTGGCAGCAGTGGA), acyl CoA oxidase ([ACOX] forward: GGATAACGGCTACCTGAAGATG; reverse: GAACAAGGTCGACAGAGGTTTAG), carnitine palmitoyltransferase 1 ([CPT-1] forward: TATGTGAGGATGCTGCTTCC; reverse: CTCGGAGCTAAGCTTGTC), acetyl-CoA carboxylase ([ACC] forward: ACCGGCTGAGTGATGGTGG; reverse: GGGAGCGCATTACAGACGG), fatty acid synthase ([FAS] forward: GCGGCTTCTGTGCCTGTTG; reverse: TCGGCAGCCCAGGCTAAGG), and sterol regulatory element-binding transcription factor 1 ([SREBP-1] forward: ACGAGCTACCCTTCGGTGA; reverse: TGTGTCTCCTGTCTCACCCC). Amplification using 40 cycles of 2 steps $\left(95^{\circ} \mathrm{C}\right.$ for $15 \mathrm{~s}$ and $60^{\circ} \mathrm{C}$ for $\left.1 \mathrm{~min}\right)$ was performed on an $\mathrm{ABI}$ Prism 7900HT sequence detection system (Foster City, CA, USA).

\section{Determination of lipolysis rate in adipose tissues}

The lipolysis rate was analyzed according to the procedure of Berger and Barnard [11]. Glycerol release from adipose tissue was expressed as the lipolysis rate. Perirenal adipose tissue was rinsed in a $0.85 \% \mathrm{NaCl}$ solution, patted dry, and weighed. A total of $0.2 \mathrm{~g}$ of adipose tissue was added to $1.0 \mathrm{ml}$ of prewarmed TES [N-tris (hydroxymethyl) methyl-2aminoethanesulfonic acid] buffer (containing $1 \mathrm{mM}$ isoproterenol) and incubated at $37^{\circ} \mathrm{C}$ for 1 hour. After incubation at $70^{\circ} \mathrm{C}$ for 10 minutes, the reaction mixture was centrifuged at $100 \mathrm{xg}$ at room temperature for $30 \mathrm{~s}$. The resulting supernatant was used to determine the glycerol concentration (Randox Diagnostic, Crumlin, UK). The lipolysis rate is expressed as $\mu \mathrm{mol}$ glycerol/g/h.

\section{Statistical evaluation}

Statistical differences among the groups were calculated by using one-way ANOVA (SAS Institute, Cary, NC, USA) and were considered to be significant at $\mathrm{p}<0.05$, as determined by Duncan's new multiplerange test.

\section{Results}

As shown in Figure 2, during electrolysis of water, the concentration of molecular hydrogen in water bath increased with time. The concentration reached up to $1041 \mathrm{ppb}$ at $8 \mathrm{~min}$ (Figure 2A). In contrast, the ORP value in water bath decreased with time (Figure 2B). The ORP value at $8 \mathrm{~min}$ was $-237 \mathrm{mv}$.

As shown in Figure 3, body weight increased in rats fed the highfat diet compared with that in rats fed the low-fat control diet after 10 weeks of feeding $(p<0.05)$. At the end of the study ( 11 weeks), daily food intake was lower and relative liver weight and relative epididymal and perirenal adipose tissue weights were higher in rats fed the high-fat diet than in rats fed the low-fat control diet $(\mathrm{p}<0.05$; Table 1). ID treatment reduced $(\mathrm{p}<0.05)$ body weight and relative perirenal adipose tissue weight in rats fed the high-fat diet. There was no significant difference ( $p>0.05$ ) in relative liver weight between the HF and HF+ID groups.

Table 2 show the plasma biochemical parameters, including glucose,

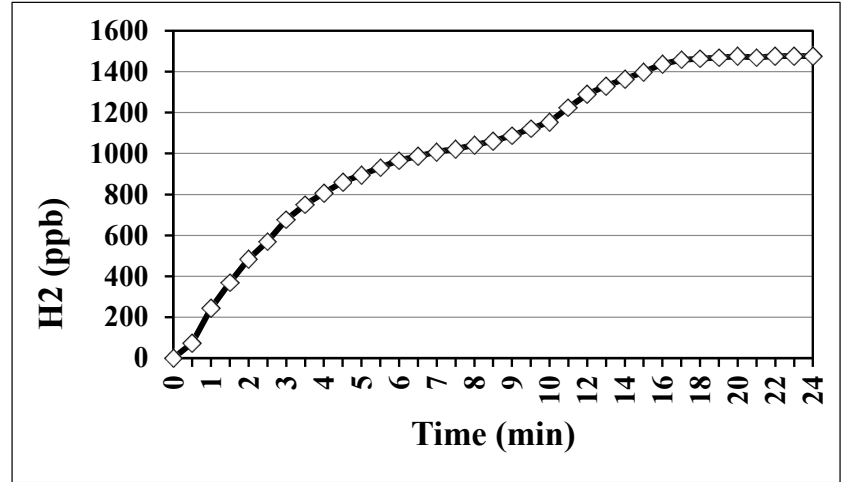

(A)

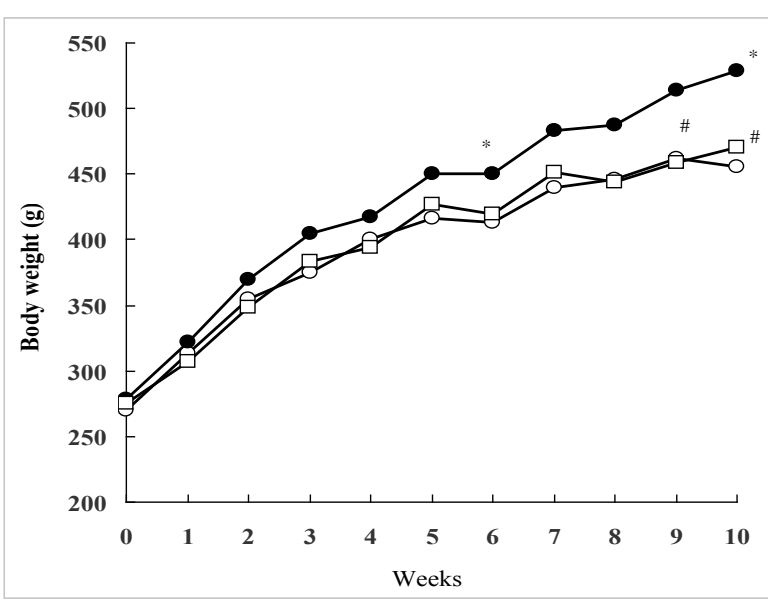

(B)

Figure 2: Changes in molecular hydrogen $\left(\mathrm{H}_{2}\right)(\mathrm{A})$ and oxidation-reduction potential (ORP) (B) in water during electrolysis. Results are expressed as the mean of 3 determinations. The detection method is described in the Methods.

insulin, total cholesterol (TC), triglyceride, HDL-cholesterol, TC/ HDL-C, ALT, and HOMA values, in rats among the groups. The plasma insulin concentration and HOMA, an index of insulin resistance, were significantly higher $(\mathrm{p}<0.05)$ in the HF group than in the control group. These results indicated that rats fed the high-fat diet developed insulin resistance. However, there were no significant differences $(p>0.05)$ in glucose, insulin, or HOMA values between the HF and HF+ID groups. No significant changes in plasma lipids (total cholesterol, HDLcholesterol, triglyceride) or ALT were observed among the groups.

Figure 4 shows the histological examination of liver with H\&E staining and hepatic triglyceride and cholesterol contents in rats. Rats fed the high-fat diet had significantly higher numbers of oil droplets (Figure 4B) and increased cholesterol (Figure 4D) and triglyceride (Figure 4E) contents $(\mathrm{p}<0.05)$ in liver than did animals fed the control diet. ID treatment reduced oil droplet numbers (Figure 4C) and triglyceride and cholesterol contents in rats fed the high-fat diet $(\mathrm{p}<0.05)$.

Table 3 shows the fecal excretions of cholesterol and triglyceride in rats. Rats fed the high-fat diet had significantly higher fecal cholesterol and triglyceride levels than did rats fed the low-fat control diet $(\mathrm{p}<0.05)$. There were no significant differences in fecal triglyceride and cholesterol levels between the HF and HF+ID groups ( $p>0.05$ ).

The gene expression of PPAR- $\alpha$ SREBP-1, ACOX, CPT-1, ACC, and 


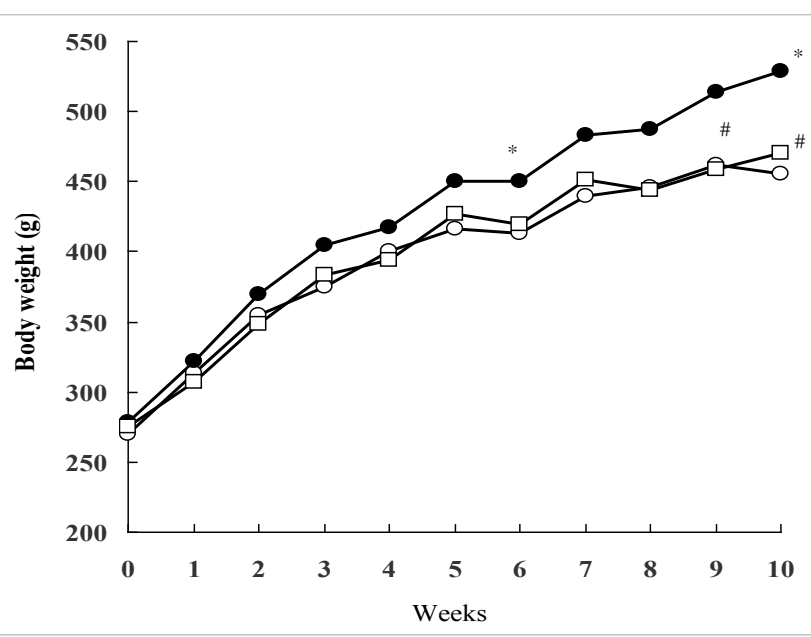

Figure 3: Effects of ID treatment on body weight gain in rats. *Significantly different from the control group at $p<0.05$. \#Significantly different from the HF group at $\mathrm{p}<0.05$. Control: normal control diet group ( $\mathrm{O})$; HF: high-fat diet group $(\bullet) ; \mathrm{HF}+\mathrm{ID}$ : high-fat diet + ID treatment group ( $\square)$.

\begin{tabular}{|l|c|c|c|}
\hline Group & Control $(\mathbf{n}=4)$ & HF $(\mathbf{n}=6)$ & HF+ID (n=6) \\
\hline Initial body weight $(\mathrm{g})$ & $270.0 \pm 10.4$ & $279.1 \pm 15.1$ & $275.8 \pm 10.4$ \\
\hline Final body weight (g) & $442.0 \pm 47.8$ & $521.8 \pm 51.5^{*}$ & $457.6 \pm 32.4^{\#}$ \\
\hline Food intake (g/day) & $25.6 \pm 0.2$ & $21.1 \pm 0.5^{*}$ & $20.5 \pm 0.6$ \\
\hline Liver weight & $11.9 \pm 1.4$ & $18.2 \pm 1.7^{*}$ & $15.7 \pm 0.6^{\#}$ \\
\hline (g) & $2.7 \pm 0.1$ & $3.5 \pm 0.1^{*}$ & $3.4 \pm 0.2$ \\
\hline (g/100 g b.w.) & $5.5 \pm 1.9$ & $9.1 \pm 1.7^{*}$ & $7.4 \pm 1.4 \#$ \\
\hline Epididymal adipose tissue & $1.7 \pm 0.2^{*}$ & $1.6 \pm 0.3$ \\
\hline (g) & $1.2 \pm 0.4$ & $11.2 \pm 2.8^{*}$ & $6.9 \pm 1.9 \#$ \\
\hline (g/100 g b.w.) & $3.8 \pm 1.7$ & $2.1 \pm 0.5^{*}$ & $1.5 \pm 0.3 \#$ \\
\hline Perirenal adipose tissue & $0.8 \pm 0.3$ & & \\
\hline (g) & $9.3 \pm 3.5$ & $20.3 \pm 4.1^{*}$ & $14.3 \pm 3.0 \#$ \\
\hline (g/100 g b.w.) & $2.1 \pm 0.7$ & $3.9 \pm 0.6^{*}$ & $3.1 \pm 0.5 \#$ \\
\hline Total adipose tissues
\end{tabular}

a Values are mean SD of 4 to 6 rats; ${ }^{b}$ Control: control group; HF: high-fat diet group; ID: high-fat diet with ID treatment group; * Significantly different from the Control group at $p<0.05$; \# Significantly different from the HF group at $p<0.05$

Table 1: Body weight, tissue weight, and food intake in rats fed the control diet, the high-fat diet, or the high-fat diet with ID treatment ${ }^{\text {. }}$

\begin{tabular}{|l|c|c|c|}
\hline Group $^{\mathbf{b}}$ & Control $(\mathbf{n}=\mathbf{4})$ & HF $(\mathbf{n}=\mathbf{6})$ & HF+ID $(\mathbf{n}=\mathbf{6})$ \\
\hline Glucose $(\mathrm{mg} / \mathrm{dL})$ & 158.012 .6 & $186.2 \pm 51.0$ & $172.8 \pm 41.9$ \\
\hline Insulin $(\mu \mathrm{gg} / \mathrm{L})$ & $0.2 \pm 0.0$ & $0.5 \pm 0.2^{*}$ & $0.5 \pm 0.2$ \\
\hline Total cholesterol $(\mathrm{mg} / \mathrm{dL})$ & $61.5 \pm 14.4$ & 73.019 .0 & $63.5 \pm 18.5$ \\
\hline Triglyceride $(\mathrm{mg} / \mathrm{dL})$ & $72.2 \pm 27.4$ & $70.2 \pm 22.1$ & $77.8 \pm 21.8$ \\
\hline HDL-cholesterol $(\mathrm{mg} / \mathrm{dL})$ & $26.5 \pm 2.5$ & $23.3 \pm 6.7$ & $22.2 \pm 5.1$ \\
\hline TC/HDL-C & $2.3 \pm 0.3$ & $3.2 \pm 0.8$ & $2.8 \pm 0.7$ \\
\hline ALT $(\mathrm{U} / \mathrm{L})$ & $52.3 \pm 6.2$ & $48.8 \pm 13.4$ & $48.3 \pm 12.1$ \\
\hline HOMA & $15.3 \pm 2.9$ & $38.3 \pm 14.7^{*}$ & $44.3 \pm 19.5$ \\
\hline
\end{tabular}

a Values are mean \pm SD of 4 to 6 rats; ${ }^{\text {b }}$ Control: control group; HF: high-fat diet group; HF+ID: high-fat diet with ID treatment group; * Significantly different from the Control group at $p<0.05$.

Table 2: Plasma parameters in rats fed the control diet, the high-fat diet, or the high-fat diet with ID treatment ${ }^{\mathrm{a}}$.

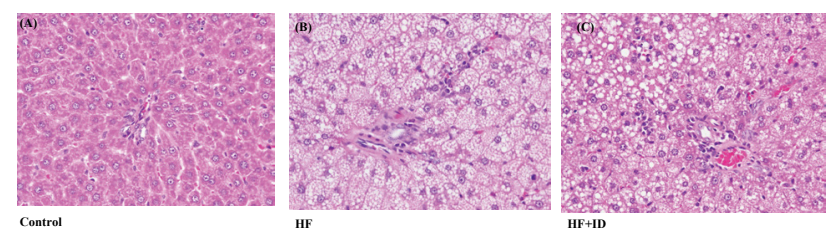

Control
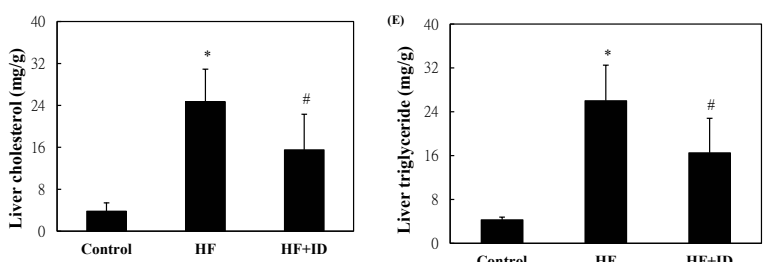

Figure 4: Histopathological alterations (A-C) and cholesterol (D) and triglyceride $(E)$ contents in livers of rats after ID treatment. (A-C) H\&E stain, $400 X$. $(D, E)$ Results are expressed as mean \pm SD for 4 to 6 rats. * Significantly different from the control group at $\mathrm{p}<0.05$. \#Significantly different from the HF group at $p<0.05$. Control: normal control diet group $(n=4) ; H F$ : high-fat diet group $(n=6)$; HF+ID: high-fat diet + ID treatment group $(n=6)$.

\begin{tabular}{|l|c|c|c|}
\hline Group $^{\mathbf{b}}$ & Control $(\mathbf{n}=\mathbf{4})$ & HF $(\mathbf{n}=6)$ & HF+ ID $(\mathbf{n}=6)$ \\
\hline Cholesterol & $5.5 \pm 0.2$ & $10.4 \pm 0.3^{*}$ & $10.4 \pm 0.2$ \\
\hline (mg/g) & $51.0 \pm 1.4$ & $143.0 \pm 3.5^{*}$ & $140.7 \pm 2.6$ \\
\hline (mg/day) & $3.9 \pm 0.1$ & $5.8 \pm 0.1^{*}$ & $5.9 \pm 0.1$ \\
\hline Triglyceride & $18.1 \pm 0.3$ & $37.6 \pm 1.2^{*}$ & $36.6 \pm 1.0$ \\
\hline$(\mathrm{mg} / \mathrm{g})$ & (mg/day)
\end{tabular}

a Values are mean \pm SD of 4 to 6 rats in each group; ${ }^{b}$ Control: control group; HF: high-fat diet group; HF+ID: high-fat diet with ID treatment group; * Significantly different from the Control group at $p<0.05$.

Table 3: Fecal lipids levels in rats fed the control diet, the high-fat diet, or the highfat diet with ID treatment ${ }^{\mathrm{a}}$.

FAS in liver is shown in Figure 5. In liver, FAS gene expression was significantly reduced in the HF group compared with the control group $(\mathrm{p}<0.05$; Figure 5F). ID treatment further reduced $(\mathrm{p}<0.05)$ ACC and FAS gene expression (Figures $5 \mathrm{E}$ and $5 \mathrm{~F}$ ). Also, ACOX and CPT-1 gene expression was significantly reduced $(\mathrm{p}<0.05)$ in rats after ID treatment (Figures 5C and 5D).

Figure 6 shows the histological examination of perirenal adipose tissue with H\&E staining and ACC and FAS gene expression in perirenal adipose tissue. No significant change in fat size was found among the groups (Figures 6A-6C). There were no significant differences in ACC or FAS mRNA levels (Figures 6D and 6E) between the HF and HF+ID groups ( $\mathrm{p}>0.05)$. A higher triglyceride content and lower lipolysis rate in perirenal adipose tissue was found in rats fed the high-fat diet $(\mathrm{p}<0.05$; Figures $6 \mathrm{~F}$ and $6 \mathrm{G})$. Notably, ID treatment reduced the triglyceride content and increased the lipolysis rate in perirenal adipose tissue $(\mathrm{p}<0.05)$.

\section{Discussion}

In this study, ID treatment was shown to reduce body weight, adiposity, and lipid accumulation in the liver of rats fed a high-fat diet. Our results also suggested that ID treatment reduced fatty acid synthesis (ACC and FAS gene expression) more significantly than fatty acid oxidation (CPT-1 and ACOX gene expression) in liver, resulting in a lower triglyceride content. A higher lipolysis rate and a lower triglyceride content were observed in perirenal adipose tissue after ID treatment, which might, at least in part, explain the anti-obesity effect. 
(A)

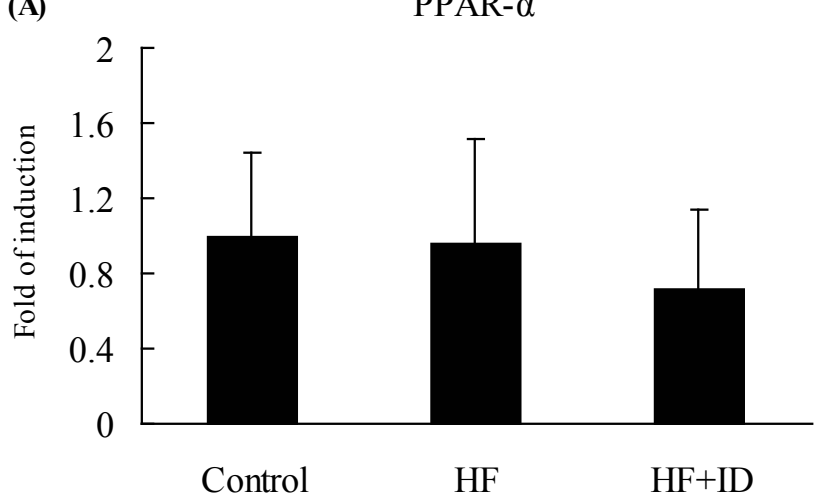

(C)

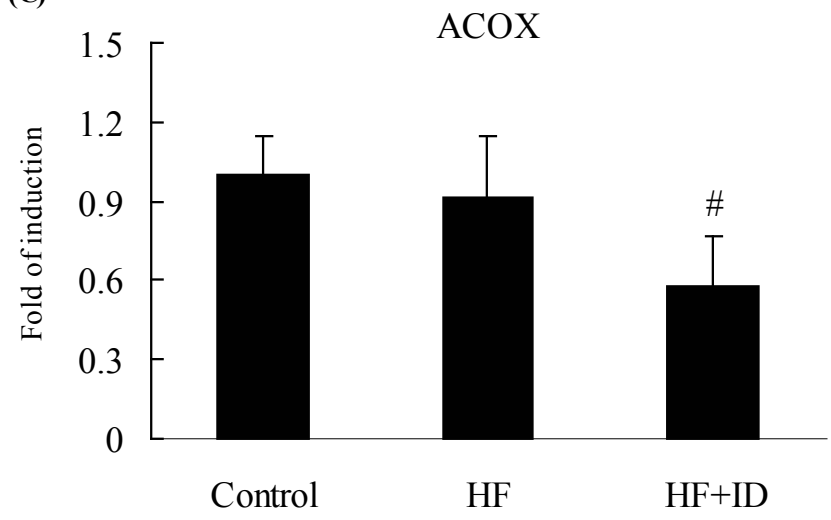

(E)

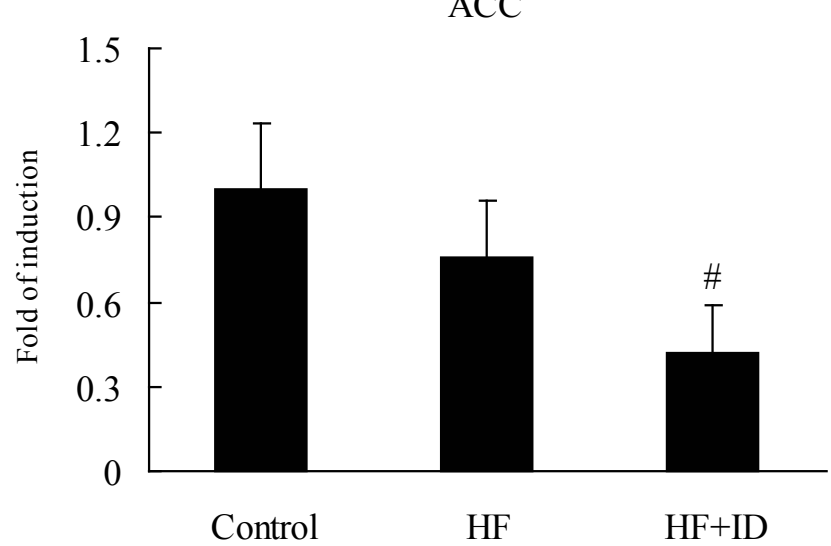

(B)

SREBP-1

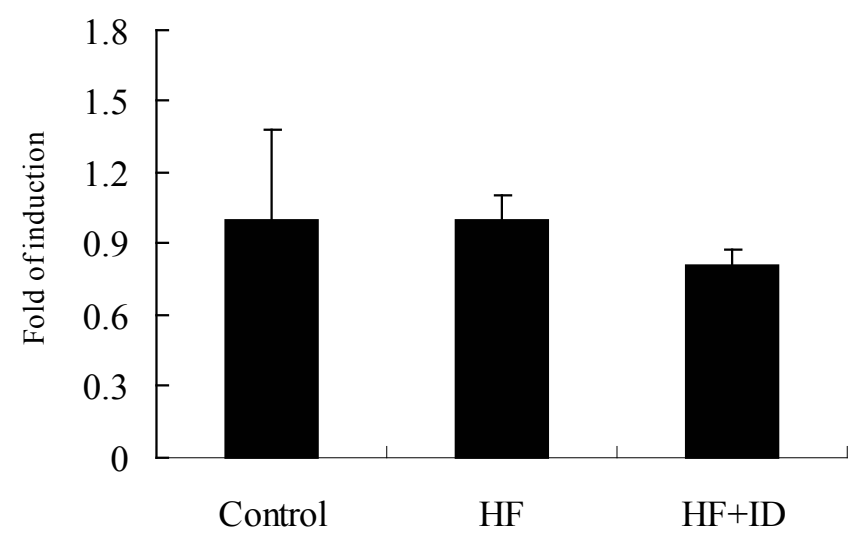

(D)

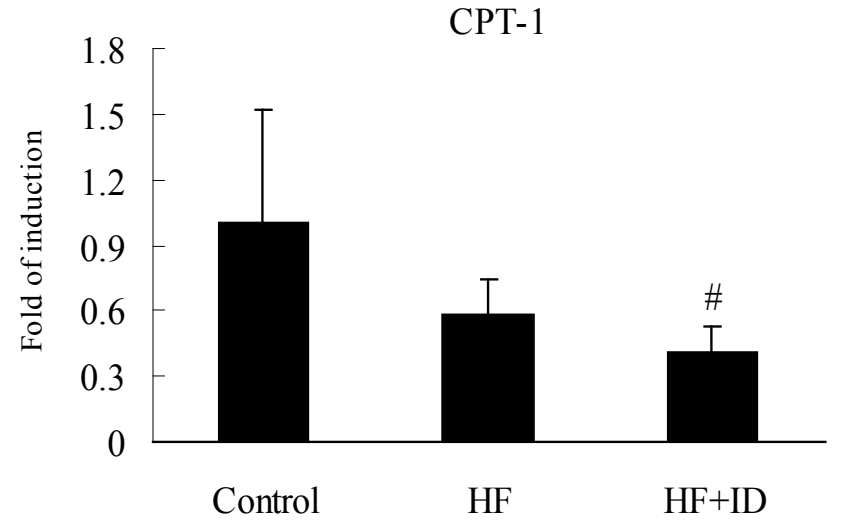

(F)

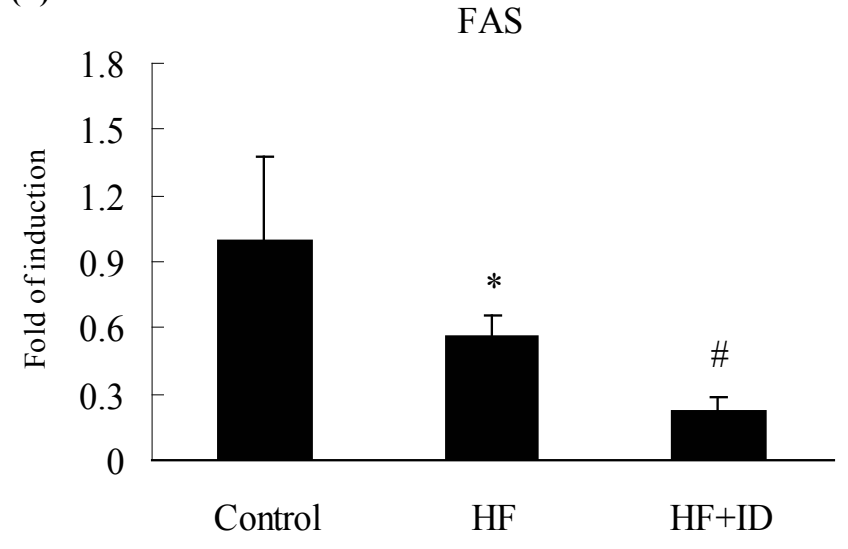

Figure 5: Changes in PPAR- $\alpha(A)$, SREBP-1 (B), ACOX (C), CPT-1 (D), ACC (E), and FAS (F) gene expression in rats. Results are expressed as mean \pm SD for 4 to 6 rats. * Significantly different from the control group at $p<0.05$. \#Significantly different from the HF group at $p<0.05$. Control: normal control diet group ( $n=4$ ); HF: high-fat diet group ( $n=6)$; HF+ID: high-fat diet + ID treatment group $(n=6)$.

In this study, regular ID treatment (3 times/week) for 11 weeks reduced the accumulation of relative adipose tissue (epididymal+perirenal; $-20.1 \%$ ) and body weight $(-12.3 \%)$ induced by the high-fat diet. However, ID treatment had no significant effect on plasma biochemical parameters including plasma lipids, glucose, insulin resistance (HOMA), and ALT. These results indicated that ID treatment can reduce adiposity without affecting other plasma biochemical parameters in this model. In addition, daily food intake and fecal lipid excretion were not changed after ID treatment, indicating that energy intake was not affected by ID treatment. In this study, ID 
(A)

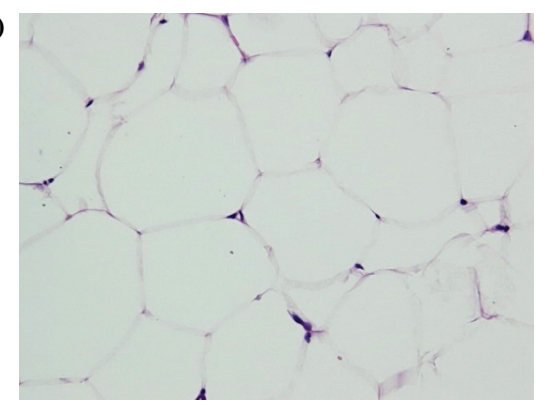

(D)

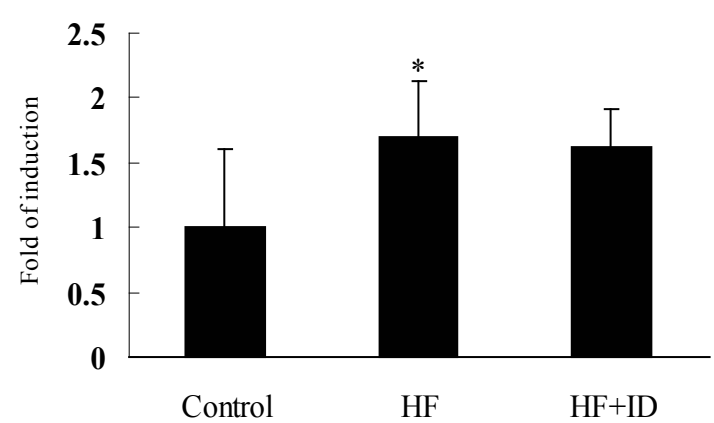

(F)

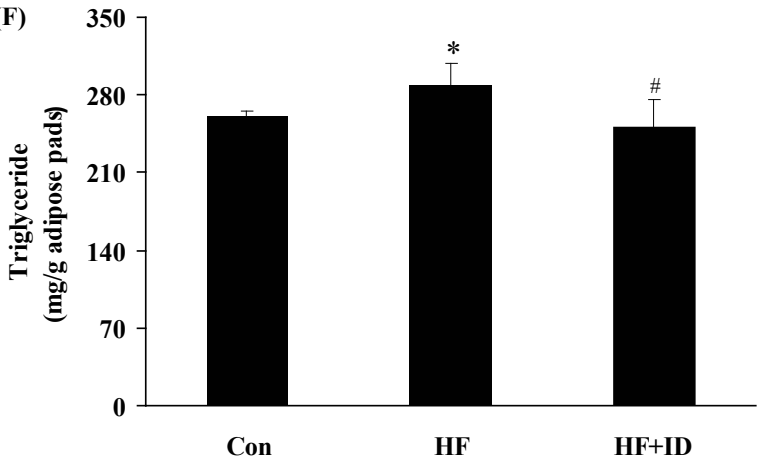

(B)

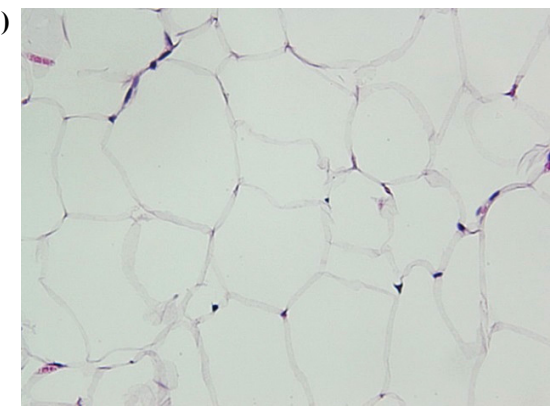

(E)
(C)

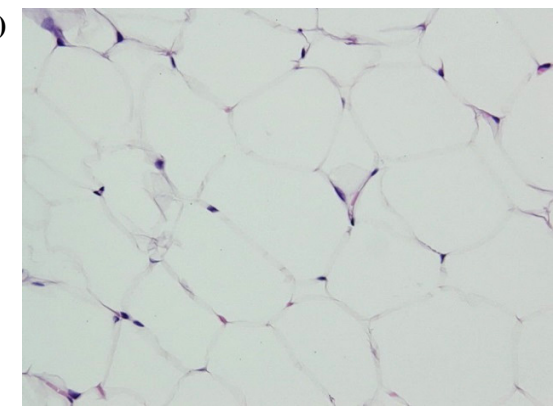

FAS
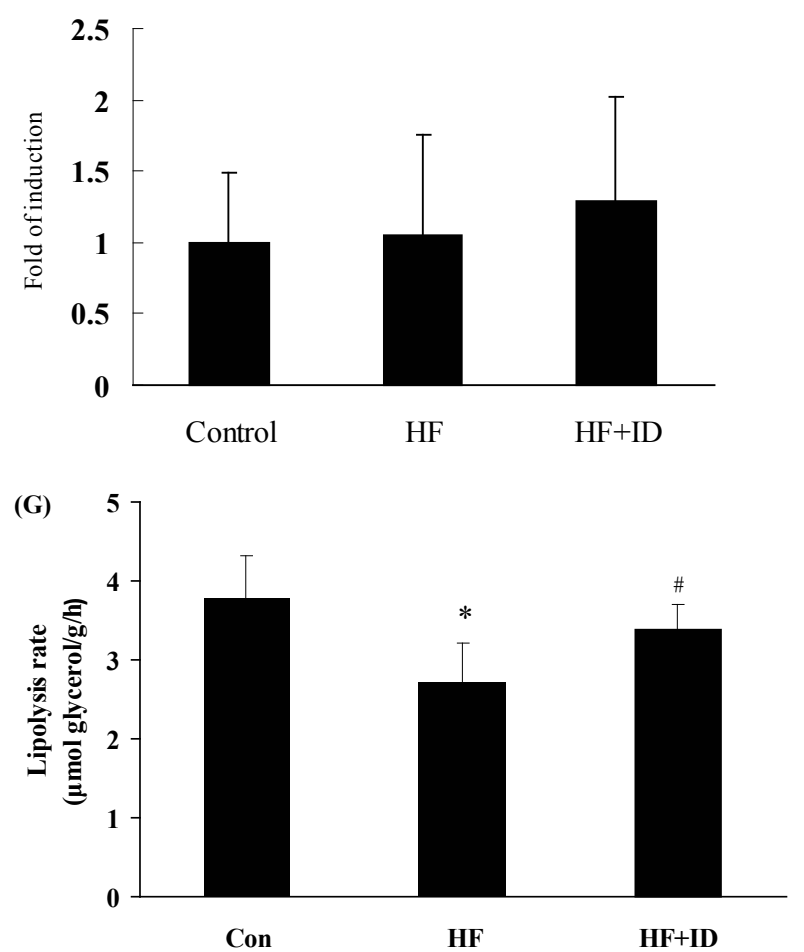

Figure 6: Histopathological examination with H\&E staining (A-C), ACC and FAS gene expression (D,E), triglyceride content (F), and lipolysis rate (G) in perirenal fat pads in rats. Results are expressed as mean \pm SD for 4 to 6 rats. *Significantly different from the control group at $p<0.05$. \#Significantly different from the HF group at $p<0.05$. Control: normal control diet group $(n=4)$; HF: high-fat diet group $(n=6)$; HF+ID: high-fat diet + ID treatment group ( $n=6)$. H\&E stain, $400 X$.

treatment had no effect on lipogenesis in perirenal adipose tissue as indicated by no change in ACC and FAS gene expression. It is therefore suggested that the reduced adiposity after ID treatment may be due to an increased lipolysis rate in perirenal adipose tissue, resulting in lower triglyceride content.

Notably, a lower hepatic triglyceride and cholesterol level were observed after ID treatment. This result indicated that ID treatment could reduce fatty liver. Geelen et al. [12] indicated that de novo fatty acid synthesis in liver could be reduced after high-fat diet feeding. In this study, rats fed the high-fat diet had reduced ACC and FAS gene expression with no changes in ACOX and CPT-1 gene expression in liver. ID treatment reduced ACC (-44.7\%), FAS (-60.7\%), CPT-1 $(-29.3 \%)$, and ACOX $(-36.2 \%)$ gene expression, indicating that ID treatment might suppress both the fatty acid synthesis and the fatty acid oxidation pathways. However, ID treatment appeared to reduce fatty acid synthesis more significantly than fatty acid oxidation. This result may explain why ID treatment can lower hepatic triglyceride content.

The human body is a conductor of electricity. In our hypothesis, during ID treatment, small positively or negatively charged molecules in the body can be moved by means of continuously transmitted microcurrents through the entire body and be attracted by the ion generator. These actions may result in a lower level of exogenous or endogenous charged molecules, thus balancing them and increasing cell detoxification. In this study, we found unknown lipids $(50 \mathrm{mg} / \mathrm{rat})$ and ammonia $(0.67 \mathrm{mg} / \mathrm{rat})$ in the bathed water after ID treatment. It is thus suggested that parts of charged molecules excreted from the body are lipophilic molecules. At physiological $\mathrm{pH}$, most of the ammonia molecules are protonated (NH4+). Therefore, ID treatment may increase detoxification by removing small charged molecules attracted by the ion generator. Finally, the neutralized molecules 
Citation: Hsien-Tsung Y (2017) Ionic Detoxification Reduces Obesity and Fatty Liver in Rats Fed a High-Fat Diet. Altern Integ Med 6: 246. doi: 10.4172/2327-5162.1000246

Page 7 of 7

were precipitated or dissolved in water with unfavorable flavors and colors (particularly in black in this study). Similar observations are also found in humans after ID treatment. Our results suggest that ID treatment may enhance cell detoxification by removing ammonia and small lipophilic charged molecules from the body. The other route of elimination of small toxic molecules during ID treatment may be possibly through forward (direct) osmosis from the microvasculature of the skin into warm water bath. Indeed, we recently found that ID treatment (bathing the rat abdominal region) reduced the hepatic activity of CYP1A1/1B1 (unpublished data), the enzyme that can be induced by various carcinogens [13]. Therefore, ID treatment may improve some metabolic disorders such as obesity and fatty liver after elimination of toxic molecules.

The other explanation for reducing adiposity and fatty liver by ID treatment may be related to the production of hydrogen-rich water (containing abundant molecular hydrogen) during the process of electrolysis (Figure 2). Hydrogen-rich water has a negative ORP value that can provide electricity to the body and lower the body's electrical potential similar to the action of grounding [4]. Molecular hydrogen is known to be the smallest molecule that can rapidly penetrate biomembranes into cells and displays antioxidant and anti-inflammation activities [14,15]. Even at low concentrations locally or throughout the whole body, molecular hydrogen can act as a signal to regulate cell function $[14,16]$. It has been shown that drinking hydrogen water can reduce obesity by enhanced fatty acid and glucose expenditure and stimulated energy metabolism [16]. Taking a hydrogen-rich water bath can also incorporate molecular hydrogen into the skin and distribute it throughout the body via blood flow within only $10 \mathrm{~min}$ [14]. Taken together, these data suggest that the reduced adiposity and fatty liver caused by regular ID treatment may be partially due to lower body electrical potential and increased molecular hydrogen uptake from the hydrogen-rich water bath.

In summary, our results showed that ID treatment may have beneficial effects on reducing adiposity through an increased lipolysis rate in adipose tissue. In addition, ID treatment may lower fatty acid synthesis and reduce fatty liver. These results may be attributed to the ability to increase the excretion of toxic or charged molecules, lower electric potential, and increase molecular hydrogen uptake during ID treatment. Further study is needed to investigate the beneficial effects of ID treatment on detoxification and metabolic disorders and the clinical applications of ID.

\section{Acknowledgments}

We are thankful for Buddha's full support. This study was financially supported by grant aid (MOST 106-2320-B-039-041-MY3) from the Ministry of Science and Technology, Taiwan.

\section{References}

1. Jamieson KS, ApSimon HM, Jamieson SS, Bell JNB, Yost MG (2007) The effects of electric fields on charged molecules and particles in individual microenvironments. Atmos Environ 41: 5224-5235.

2. Milham S (2014) Evidence that dirty electricity is causing the worldwide epidemics of obesity and diabetes. Electromagn Biol Med 33: 75-78.

3. Zheng BW, Ho WC (2013) Electromagnetic principles in cardiovascular disease development. Taiwan Crit Care Med 14: 57-60.

4. Chevalier G, Sinatra ST, Oschman JL, Sokal K, Sokal P (2012) Earthing: Health implications of reconnecting the human body to the Earth's surface electrons. J Environ Public Health 2012: 291541.

5. Oschman JL, Chevalier G, Brown R (2015) The effects of grounding (earthing) on inflammation, the immune response, wound healing, and prevention and treatment of chronic inflammatory and autoimmune diseases. J Inflamm Res 8: 83-96.

6. Ghibaudi L, Cook J, Farley C, van Heek M, Hwa JJ (2002) Fat intake affects adiposity, comorbidity factors, and energy metabolism of Sprague-Dawley rats. Obes Res 10: 956-963.

7. Drury AR, Wallington EA (1980) Carleton's Histological Techniques. 5th ed., Oxford University Press, London, UK.

8. Matthews DR, Hosker JP, Rudenski AS, Naylor BA, Treacher DF, et al. (1985) Homeostasis model assessment: Insulin resistance and beta-cell function from fasting plasma glucose and insulin concentrations in man. Diabetologia 28 412-419.

9. Folch J, Lees M, Sloane-Stanley GM (1957) A purification of total lipid from animal tissue. J Biol Chem 226: 497-509.

10. Carlson SE, Goldfarb S (1977) A sensitive enzymatic method for determination of free and esterified tissue cholesterol. Clin Chim Acta 79: 575-582.

11. Berger JJ, Barnard RJ (1999) Effect of diet on fat cell size and hormonesensitive lipase activity. J Appl Physiol 87: 227-232.

12. Geelen SN, Blázquez C, Geelen MJ, Sloet van Oldruitenborgh-Oosterbaan MM, Beynen AC (2008) High fat intake lowers hepatic fatty acid synthesis and raises fatty acid oxidation in aerobic muscle in Shetland ponies. $\mathrm{Br} \mathrm{J}$ Nutr 86 $31-36$.

13. Shimada T, Oda Y, Gillam EM, Guengerich FP, Inoue K (2001) Metabolic activation of polycyclic aromatic hydrocarbons and other procarcinogens by cytochromes P450 1A1 and P450 1B1 allelic variants and other human cytochromes P450 in Salmonella typhimurium NM2009. Drug Metab Dispos 29: 1176-1182.

14. Ichihara M, Sobue S, Ito M, Ito M, Hirayama M, et al. (2015) Beneficial biological effects and the underlying mechanisms of molecular hydrogen-comprehensive review of 321 original articles. Med Gas Res 5: 12

15. Ohta S (2012) Molecular hydrogen is a novel antioxidant to efficiently reduce oxidative stress with potential for the improvement of mitochondrial diseases. Biochim Biophys Acta 1820: 586-594.

16. Kamimura N, Nishimaki K, Ohsawa I, Ohta S (2011) Molecular hydrogen improves obesity and diabetes by inducing hepatic FGF21 and stimulating energy metabolism in db/db mice. Obesity (Silver Spring) 19: 1396-1403. 